\title{
Examining the T Tauri system with SPHERE ${ }^{\star}, \star \star$
}

\author{
Gergely Csépány ${ }^{1,2}$, Mario van den Ancker ${ }^{1}$, Péter Ábrahám², Wolfgang Brandner ${ }^{3}$, and Felix Hormuth ${ }^{3}$ \\ 1 European Southern Observatory, Karl-Schwarzschild-Str. 2, 85748 Garching bei München, Germany \\ e-mail: gcsepany@eso.org \\ 2 Konkoly Observatory of the Research Centre for Astronomy and Earth Sciences, Hungarian Academy of Sciences, \\ Konkoly Thege Miklós út 15-17, 1121 Budapest, Hungary \\ 3 Max-Planck-Institut für Astronomie, Königstuhl 17, 69117 Heidelberg, Germany
}

Received 27 March 2015 / Accepted 19 May 2015

\section{ABSTRACT}

\begin{abstract}
Context. The prototypical low-mass young stellar object, T Tauri, is a well-studied multiple system with at least three components. Aims. We aim to explore the T Tau system with the highest spatial resolution, study the time evolution of the known components, and re-determine the orbital parameters of the stars.

Methods. Near-infrared classical imaging and integral field spectrograph observations were obtained during the Science Verification of SPHERE, the new high-contrast imaging facility at the VLT. The obtained FWHM of the primary star varies between $0.050^{\prime \prime}$ and $0.059^{\prime \prime}$, making these the highest spatial resolution near-infrared images of the T Tauri system obtained to date.

Results. Our near-infrared images confirm the presence of extended emission south of $\mathrm{T}$ Tau Sa, reported in the literature. New narrow-band images show, for the first time, that this feature shows strong emission in both the $\mathrm{Br}-\gamma$ and $\mathrm{H}_{2} 1-0 \mathrm{~S}(1)$ lines. Broadband imaging at $2.27 \mu \mathrm{m}$ shows that T Tau Sa is 0.92 mag brighter than T Tau Sb, which is in contrast to observations from Jan. 2014 (when T Tau Sa was fainter than Sb), and demonstrates that T Tau Sa has entered a new period of high variability. The newly obtained astrometric positions of T Tau Sa and Sb agree with orbital fits from previous works. The orbit of T Tau S (the centre of gravity of Sa and $\mathrm{Sb}$ ) around $\mathrm{T}$ Tau $\mathrm{N}$ is poorly constrained by the available observations and can be fit with a range of orbits ranging from a nearly circular orbit with a period of 475 years to highly eccentric orbits with periods up to $2.7 \times 10^{4}$ years. We also detected a feature south of T Tau N, at a distance of $144 \pm 3$ mas, which shows the properties of a new companion.
\end{abstract}

Key words. stars: variables: T Tauri, Herbig Ae/Be - stars: pre-main sequence - stars: evolution - binaries: visual techniques: high angular resolution - instrumentation: adaptive optics

\section{Introduction}

T Tauri (HD 284419), initially considered to be the prototype of a class of low-mass, pre-main sequence stars, is now known to be a complex young system of at least three stellar components ( $\mathrm{T}$ Tau N, and the close binary system $\mathrm{T}$ Tau $\mathrm{Sa} / \mathrm{Sb}$ ), as well as of jets and outflows. Whereas $\mathrm{T}$ Tau $\mathrm{N}$ suffers little extinction $\left(A_{V}=1.46\right.$; White \& Ghez 2001), $\mathrm{T}$ Tau Sb is hidden behind about 15 mag of extinction, while T Tau Sa appears to be even more obscured (van den Ancker et al. 1999; van Boekel et al. 2010; Duchêne et al. 2005).

Orbital motion of $\mathrm{Sa}$ and $\mathrm{Sb}$ has been measured over the last 16 years, yielding a well-constrained orbital model for Sa$\mathrm{Sb}$ with a semi-major axis of 89 mas and a period of 29 years (Schaefer et al. 2014). Using the orbital data, the mass of T Tau Sa was shown to be 1.9-2.3 $M_{\odot}$ and the mass of Sb is between 0.7 and $0.9 M_{\odot}$ (Duchêne et al. 2006; Köhler et al. 2008). The orbital motion of the Sa-Sb system around T Tau N is less well constrained, with initial fits yielding orbital periods between 475 and 23000 years, and a total system mass between 4.5 and 5.6 $M_{\odot}$ (Johnston et al. 2004; Köhler et al. 2008).

Millimetre interferometry first revealed the presence of a circumstellar disc around the optically dominant component

\footnotetext{
* Based on observations made at the La Silla Paranal Observatory under programme ID 60.A-9363(A) and 60.A-9364(A).

$\star \star$ Figures 3-5 are available in electronic form at http://www . aanda.org
}

T Tau N (Hogerheijde et al. 1997; Akeson et al. 1998). The presence of CO absorption in the spectrum of T Tau Sa suggests the presence of a circumstellar disc viewed nearly edge-on around this source as well (Duchêne et al. 2005). In the high-resolution images, van Boekel et al. (2010) found circumstellar emission around T Tau Sa in the $K$-band on scales of 5 to $20-30 \mathrm{AU}$. It is as of yet unclear whether this emission can be directly associated with the tentative edge-on disc of T Tau Sa. All three components of the T Tau system are actively accreting and are spatially unresolved hydrogen recombination line emitters (Kasper et al. 2002; Gustafsson et al. 2010).

In this paper we present new infrared images and spectroscopy of the T Tau system obtained with SPHERE at the VLT. At small scales, these new images present the highest contrast data obtained of T Tau so far. We discuss the orbital and photometric evolution of the known components of $\mathrm{T}$ Tau and present evidence for the presence of a new tentative companion south of T Tau N.

\section{Observations and data analysis}

Observations of the T Tau system were acquired during the Science Verification of SPHERE ${ }^{1}$, the newly installed extreme adaptive optics facility at the VLT. The first set of observations

\footnotetext{
1 http://www.eso.org/sci/facilities/paranal/ instruments/sphere.html
} 


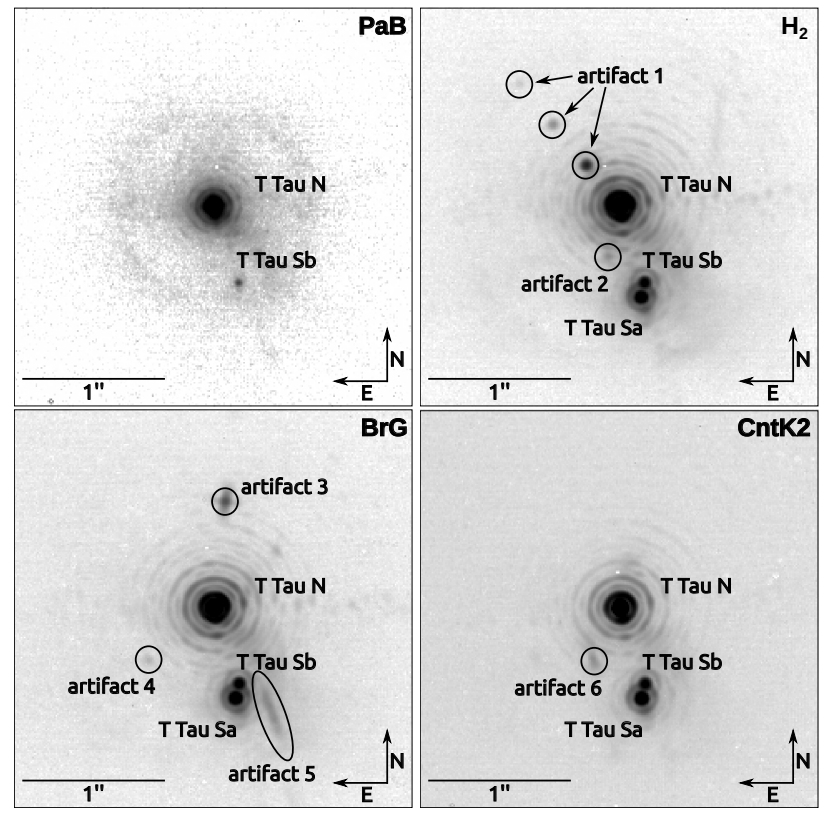

Fig. 1. Classical images of the T Tauri system in different filters with logarithmic intensity scaling. From top left to bottom right: $\mathrm{Pa}-\beta$ (1.281 $\mu \mathrm{m}), \mathrm{H}_{2}(2.128 \mu \mathrm{m}), \mathrm{Br}-\gamma(2.163 \mu \mathrm{m})$, and CntK2 $(2.270 \mu \mathrm{m})$.

was obtained on Dec. 9th, 2014, when SPHERE was operated in the IRDIS classical imaging mode with the ContJ $(1.216 \mu \mathrm{m})$, $\mathrm{Pa}-\beta(1.281 \mu \mathrm{m}), \mathrm{H}_{2}(2.128 \mu \mathrm{m}), \mathrm{Br}-\gamma(2.163 \mu \mathrm{m})$, and CntK2 $(2.270 \mu \mathrm{m})$ filters. Calibration frames were acquired for the geometrical distortion map, which we used during the data processing to ensure astrometric precision. A second set of observations was acquired on Jan. 22nd, 2015, when SPHERE was operated in the IRDIFS mode with a 155 milli-arcsecond (mas) diameter apodized Lyot coronagraph, offering simultaneous Integral Field Spectrograph (IFS) observations from $0.95-1.65 \mu \mathrm{m}$, and imaging in the $K \mathrm{~s}(2.181 \mu \mathrm{m})$ filter. We also obtained observations with the star offset from the coronagraphic mask to be able to measure total fluxes. A point spread function (PSF) reference star (TYC 1290-457-1) was observed right after the IRDIFS observation of T Tau, at similar airmass and atmospheric conditions as the science target.

The obtained data sets were reduced with the pre-release version 0.14.0-2 of the SPHERE pipeline. Apart from the usual steps of bias subtraction, flat-fielding, and wavelength calibration, the pipeline also includes a correction for geometric distortions. Post-processing of the data was done using the FITSH software package (Pál 2012) to sum the data resulting from the two IRDIS channels. The achieved FWHM of the diffractionlimited core of T Tau N varies between 0.050" and 0.059" (from ContJ to CntK2), making these the highest spatial resolution near-infrared images of the $\mathrm{T}$ Tau system obtained to date. The classical images are shown in Fig. 1 . The $\mathrm{H}_{2}, \mathrm{Br}-\gamma$, and CntK2 filters suffer from a number of known ghosts that can be seen close to bright stars. Figure 2 features the IFS images taken with the coronagraph, in which a cross-like pattern can be seen that is caused by reflections from the wires on which the coronagraphic mask is suspended. The longer wavelength frames in the IFS observation suffer from a flat-fielding error pattern; either the flat fields were inadequate or the pre-release version of the pipeline failed to properly reduce the data. We do not perform precise astrometry and photometry in those frames, and only use them to determine the spectrum with a larger aperture to compensate for the error pattern.
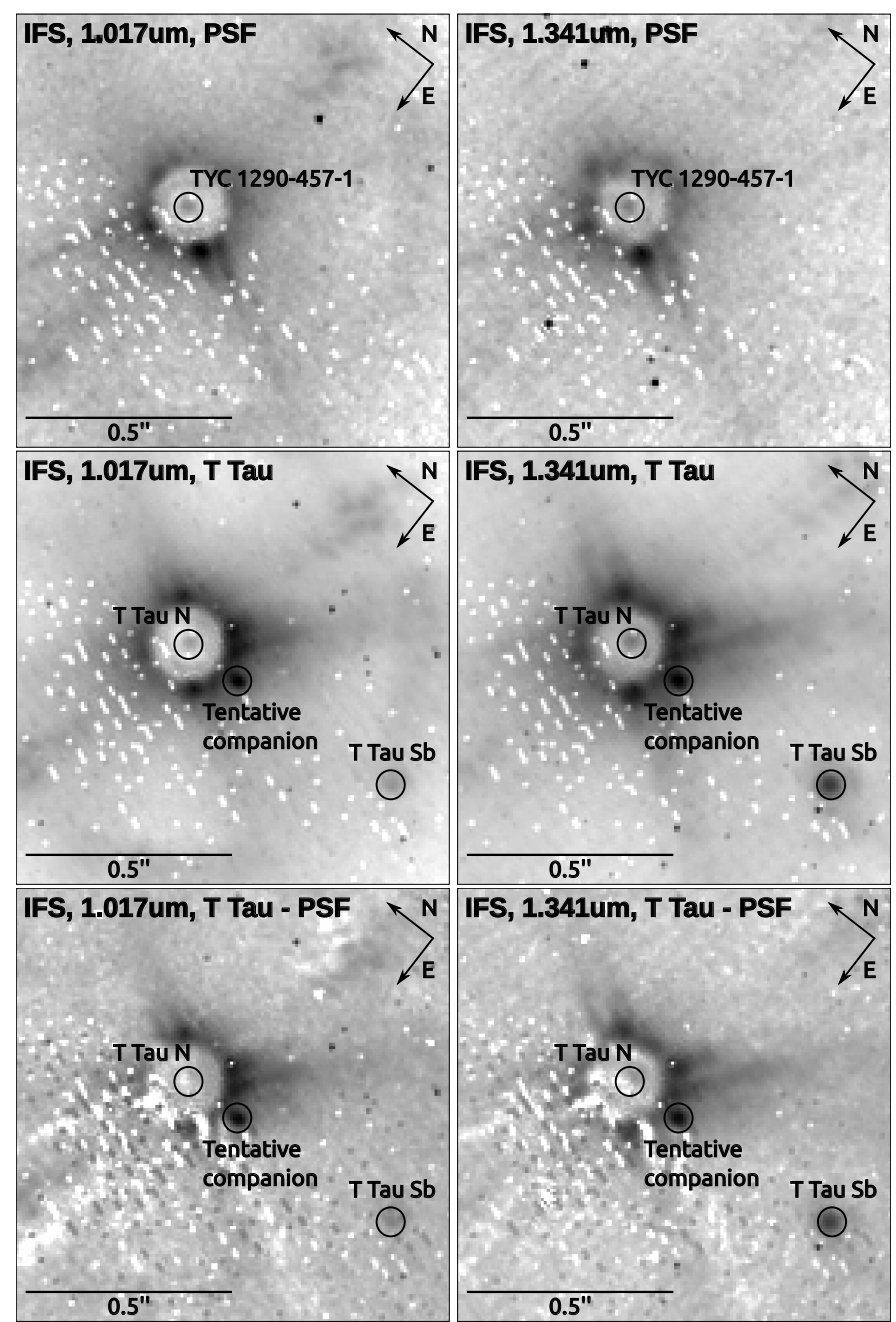

Fig. 2. The T Tauri system as seen at two different wavelengths in the IFS observation. Top: PSF reference star (TYC 1290-457-1), middle: T Tauri system, bottom: T Tauri with the PSF reference subtracted.

Positions and fluxes (both relative to $\mathrm{T}$ Tau N) of the stellar components $\mathrm{T}$ Tau Sa and $\mathrm{Sb}$ were measured and are listed in Table 1. Relative fluxes in this Table were computed using aperture photometry, with the aperture radius taken equal to the radius of the Airy disc (73.5 mas). The listed uncertainties of the stellar positions and angles include the error of the centroid fitting, the pixel-scale conversion uncertainty, and relative atmospheric diffraction. The latter error contribution is calculated by assuming the maximum relative shift in position of the components diffraction in the used filter.

\section{Results}

\subsection{Classical imaging}

The two shortest wavelength images (ContJ and $\mathrm{Pa}-\beta$ ) show the primary (T Tau N) and one of the southern components ( $\mathrm{T}$ Tau $\mathrm{Sb}$ ). In addition, some extended emission may be present at low intensities. In the longer wavelength images $\left(\mathrm{H}_{2}, \mathrm{Br}-\gamma\right.$ and CntK2), the second known southern component (T Tau Sa) is also visible. In all images, the stellar sources $\mathrm{T} \mathrm{Tau} \mathrm{N}, \mathrm{Sa}$, and $\mathrm{Sb}$ appear indistinguishable from a point source.

We calculated observational $3 \sigma$ detection limits in the different filters from our data and these are shown in Fig. 4 by calculating the magnitude of a hypothetical source that is hidden in the background noise at the given location. The bumps in the plots 
Table 1. Positions and magnitude differences (relative to T Tauri N) of the stellar components.

\begin{tabular}{llcll}
\hline \hline Object & Filter & Sep. (mas) & PA $\left(^{\circ}\right)$ & Magn. diff. \\
\hline T Tau Sa & $\mathrm{H}_{2}$ & $688 \pm 3$ & $193.4 \pm 0.2$ & $2.41 \pm 0.01$ \\
& $\mathrm{Br}-\gamma$ & $686 \pm 3$ & $193.4 \pm 0.2$ & $2.26 \pm 0.01$ \\
& $\mathrm{CntK} 2$ & $685 \pm 3$ & $193.3 \pm 0.2$ & $1.95 \pm 0.01$ \\
& $\mathrm{Ks}$ & $687 \pm 15$ & $193.1 \pm 1.0$ & $1.82 \pm 0.03$ \\
T Tau Sb & $\mathrm{ContJ}$ & $589 \pm 6$ & $198.2 \pm 0.4$ & $6.14 \pm 0.12$ \\
& $\mathrm{~Pa}-\beta$ & $590 \pm 6$ & $198.2 \pm 0.4$ & $5.77 \pm 0.09$ \\
& $\mathrm{H}_{2}$ & $595 \pm 3$ & $197.9 \pm 0.3$ & $3.15 \pm 0.01$ \\
& $\mathrm{Br}-\gamma$ & $593 \pm 3$ & $198.0 \pm 0.3$ & $3.09 \pm 0.02$ \\
& $\mathrm{CntK} 2$ & $592 \pm 3$ & $197.9 \pm 0.3$ & $2.87 \pm 0.02$ \\
& $\mathrm{Ks}$ & $589 \pm 15$ & $197.7 \pm 1.3$ & $2.58 \pm 0.05$ \\
Tentative & $1.02 \mu \mathrm{m}$ & $140.9 \pm 2.7$ & $199.0 \pm 2.8$ & $4.96 \pm 0.30$ \\
companion & $1.19 \mu \mathrm{m}$ & $143.7 \pm 2.5$ & $198.0 \pm 1.6$ & $4.39 \pm 0.19$ \\
(IFS obs.) & $1.34 \mu \mathrm{m}$ & $145.8 \pm 3.1$ & $197.2 \pm 2.0$ & $4.37 \pm 0.30$ \\
\hline
\end{tabular}

at $\approx 250$ and $\approx 600$ mas are the result of the Airy rings. We do not detect the tentative northern component, denoted as T Tau (O') by Nisenson et al. (1985) (and later also observed by Maihara \& Kataza 1991). Our upper limits show that this tentative component, if it exists, must be at least 4.7 mag fainter than $\mathrm{T}$ Tau $\mathrm{N}$ over the entire $1.2-2.2 \mu \mathrm{m}$ range covered by our observations.

In our CntK2 $(2.27 \mu \mathrm{m})$ image, T Tau Sa appears distinctly brighter $(0.92 \mathrm{mag}$, Table 1$)$ than T Tau Sb. This is in marked contrast to the latest $K$-band brightness ratio measurements, obtained in January 2014, in which Sa was about 0.8 mag fainter than Sb (Schaefer et al. 2014). This variability of T Tau Sa of $1.7 \mathrm{mag}$ in less than a year is similar to the photometric behaviour shown prior to 2006 (Beck et al. 2004; Duchêne et al. 2005), and demonstrates that the period of lesser variability reported from 2006-2013 (van Boekel et al. 2010; Schaefer et al. 2014) has come to an end.

In addition to the continuum filters, our data set includes measurements in several narrow-band filters centred on wellknown emission lines ( $\mathrm{Pa}-\beta, \mathrm{Br}-\gamma$ and the $\mathrm{H}_{2} 1-0 \mathrm{~S}(1)$ line). From the brightness ratio of $\mathrm{Br}-\gamma$ to the nearby $\mathrm{CntK} 2$ filter, we conclude that $\mathrm{T}$ Tau $\mathrm{N}$ (brightness ratio 1.52) shows strong $\mathrm{Br}-$ $\gamma$ emission, most likely linked to the accretion onto the central star. Measurements for $\mathrm{Sa}$ and $\mathrm{Sb}$ (brightness ratios of 1.14 and 1.22 , respectively) are inconclusive, although we note that $\mathrm{Br}-\gamma$ emission has previously been detected from both these components (Gustafsson et al. 2010).

For $\mathrm{H}_{2}$, the brightness ratio $\mathrm{H} 2 / \mathrm{CntK} 2$ is very high (1.90) for $\mathrm{T}$ Tau $\mathrm{N}$, demonstrating the presence of strong $\mathrm{H}_{2} 1-0$ $\mathrm{S}(1)$ emission close to this component. Previous studies found $\mathrm{H}_{2}$ emission around T Tau N and S (Kasper et al. 2002; Beck et al. 2004; Gustafsson et al. 2010), which our observations also confirm. Our CntK2, $\mathrm{Br}-\gamma$, and $\mathrm{H}_{2}$ images (Fig. 5) show extended emission at a peak flux of $6 \sigma$ above background level south of T Tau Sa, first described by van Boekel et al. (2010, Fig. 5). The ratio of the flux in the $\mathrm{H}_{2}$ and $\mathrm{Br}-\gamma$ filters to that in CntK2 (1.78 and 1.53, respectively) is high, demonstrating that this feature possesses emission in the $\mathrm{Br}-\gamma$ and the $\mathrm{H}_{2}$ 1-0 $\mathrm{S}(1)$ lines.

\subsection{Integral field spectrograph}

Our IFS data show a point-like feature south of $\mathrm{T}$ Tau N, which we identify as a tentative new component in the system. This new companion is shown in Fig. 2, which shows the PSF reference star TYC 1290-457-1, the T Tau system, and the T Tau system with the PSF reference star subtracted at two different wavelengths (1.017 and $1.341 \mu \mathrm{m})$. The astrometric and photometric measurements of the new companion are computed with a $0.1 \mu \mathrm{m}$ binning, and are listed in Table 1 .

Although several artefacts can be seen in the IFS data shown in Fig. 2, the feature we label here as a tentative new companion is unique in several aspects: (1) the tentative companion is only seen in the T Tau IFS data; no similar feature is present in the PSF data; (2) the tentative companion has the same position at the different wavelengths in the IFS data cube, excluding the possibility that this could be a speckle from the AO system; and (3) the spectrum of the tentative component is much redder than the spectrum of $\mathrm{T}$ Tau $\mathrm{N}$ (the spectral slope in $F_{v}$ between $0.97-1.30 \mu \mathrm{m}$ is 1.3 for the companion and 0.9 for T Tau $\mathrm{N}$ ), excluding the possibility that this could be a reflection from $\mathrm{T}$ Tau $\mathrm{N}$ in the instrument's optics.

Close inspection of the IRDIS images in Fig. 1 also reveals a slightly extended point-like source just south of $\mathrm{T}$ Tau N, although its distance is close to the first Airy ring. As we do not have a PSF reference observation for the IRDIS frames, we cannot perform precise measurements in those frames and we rely on the IFS frames to determine the companion's properties. The tentative companion is not detected in the IRDIS $K$ s images and is not found in the $K \mathrm{p}$ - and $H$-band images made by Keck/NIRC2 (Schaefer et al. 2014; Duchêne et al. 2006). The IRDIS images have an observational contrast limit of $\approx 3.3 \mathrm{mag}$ at 140 mas, one magnitude less than the brightness of the tentative companion, and the Keck/NIRC2 images have a contrast limit of $\approx 1.0$ at the same position. Therefore it is likely that the tentative companion is lost in the sky background in these images.

The absolute magnitude of the tentative companion can be calculated using the 2MASS brightness of T Tau N, and the distance modulus derived by Loinard et al. (2005), yielding $J_{\mathrm{mag}}=$ $6.0 \pm 0.4$. Assuming an age of the system of $2 \mathrm{Myr}$ (White \& Ghez 2001), and using the evolutionary tracks of Baraffe et al. (1998), this absolute magnitude is compatible with a stellar or sub-stellar companion with mass between 0.06 and $0.15 M_{\odot}$.

\subsection{Astrometry}

We compared our new astrometric measurements of the relative positions of T Tau N, Sa, and Sb with all available astrometry of the T Tau system from the literature (Fig. 3). The literature contains (mostly recent) astrometric measurements in which Sa and $\mathrm{Sb}$ have been spatially resolved, and (mainly older) measurements in which only one southern component was detected. In our IFS observations T Tau Sa becomes visible at wavelengths longer than $1.4 \mu \mathrm{m}$, consistent with the IRDIS images, where $\mathrm{T}$ Tau Sa is only visible in the longer wavelength observations. Therefore, we can safely assume that any observation at wavelengths shorter than $1.4 \mu \mathrm{m}$, which did not resolve the southern components, has in fact observed $\mathrm{T}$ Tau Sb. We thus interpret those measurements as reflecting the position of $\mathrm{T} \mathrm{Tau} \mathrm{Sb}$. We also know that the luminosity of $\mathrm{T} \mathrm{Tau} \mathrm{Sa} \mathrm{is} \mathrm{comparable} \mathrm{to}$ $\mathrm{T}$ Tau Sb in the $K$-band and is highly variable (van Boekel et al. 2010; Schaefer et al. 2014). This makes it impossible to use unresolved $K$-band observations to determine the exact position of $\mathrm{Sa}$ and $\mathrm{Sb}$. We therefore omit from our analysis any literature observations in the $K$-band, in which $\mathrm{Sa}$ and $\mathrm{Sb}$ have not been resolved.

As can be seen from Fig. 3, we detected significant orbital motion of the Sa-Sb binary around $\mathrm{T} \mathrm{Tau} \mathrm{N}$, and in the Sa-Sb system itself. The positions are relative to either T Tau N (left) or T Tau Sa (right). We labelled the observations for the literature 
Table 2. New orbital parameters for T Tau N-S.

\begin{tabular}{lc}
\hline \hline Parameter & Range \\
\hline$a\left(^{\prime \prime}\right)$ & $0.57-16.8$ \\
$a(\mathrm{AU})$ & $84-2465$ \\
Eccentricity & $0.10-0.98$ \\
Inclination $\left(^{\circ}\right)$ & $0.6-78.1$ \\
$\omega\left(^{\circ}\right)$ & $1.2-178.0$ \\
$\Omega\left({ }^{\circ}\right)$ & $14.1-176.7$ \\
Period (years) & $475-27000$ \\
$T_{\text {periastron }}$ (years) & $1400-7200$ \\
\hline
\end{tabular}

source. The unresolved observations are shown in light grey for the $K$-band, and blue for the $H$-band. Both are corrected for the motion of $\mathrm{T}$ Tau $\mathrm{Sb}$ around $\mathrm{T}$ Tau Sa, using the orbital parameters from Schaefer et al. (2014). The $H$-band data align with the resolved observations, whereas the $K$-band data (which are affected by the variable brightness ratio of $\mathrm{Sa} / \mathrm{Sb}$ ) seem to deviate.

The corrected $H$-band points and the epochs from resolved observations miss the orbital fit of the T Tau S around T Tau N (the centre of gravity of Sa and Sb) from Köhler et al. (2008), clearly showing that this previously derived orbit does not correctly take the orbital motion of $\mathrm{T}$ Tau $\mathrm{Sb}$ around $\mathrm{Sa}$ into account. Therefore, we searched for new orbital fits. We have excluded some observations from the orbital fit as their uncertainties were too low to acquire a $\chi_{v}^{2}<1$ fit, as shown in Fig. 3. There are also radio observations of this system, which list astrometric positions for presumably T Tau Sb, see Johnston et al. (2003) and Smith et al. (2003). However, even after correcting for the orbital motion of $\mathrm{Sb}$ around $\mathrm{Sa}$, the radio observations do not align with the optical observations, and instead are grouped with the unresolved $K$-band observations. This suggests that the origin of the radio emission may be more complex, and it may not originate only from $\mathrm{T}$ Tau $\mathrm{Sb}$, therefore, we omitted them from the plot and the orbital fit.

A random grid search of the parameter space yields a wide range of possible solutions for the orbit of $T$ Tau $S$ around $\mathrm{T}$ Tau N. The possible range of orbital parameters is shown in Table 2. Some of the parameters listed in this table exclude the orbital solution derived by Köhler et al. (2008). This is a natural consequence of the clear differences in our fits, as can seen from Fig. 3. Assuming the same measurement precision that we achieved with SPHERE, a unique solution for the orbit should be possible with new data obtained around 2027-28. Regarding the orbital motion of $\mathrm{Sa}$ around $\mathrm{Sb}$, our fit is essentially identical to the orbital fit by Schaefer et al. (2014).

\section{Summary and conclusions}

In the new SPHERE data on the T Tauri system presented here, two components, $\mathrm{T}$ Tau $\mathrm{N}$ and $\mathrm{Sb}$ are visible in all classical images, while Sa only becomes visible at wavelengths longer than $1.4 \mu \mathrm{m}$. All components appear indistinguishable from a point source. We detected a new tentative companion south of T Tau N, at a position of $144 \pm 3$ mas, PA: $197 \pm 2^{\circ}$, relative to T Tau N. This companion is not present in the PSF reference observation, and its spectrum differ from the other stellar companions in the system. If this is a new stellar or sub-stellar source within the system, we estimate its mass to be between 0.06 and $0.15 M_{\odot}$.

We compared new astrometric measurements of the relative positions of $\mathrm{T}$ Tau N, Sa and $\mathrm{Sb}$ with astrometric data from the literature. The astrometric epochs agree with the orbital fit from Schaefer et al. (2014) for the expected orbital motion of $\mathrm{Sb}$ around $\mathrm{Sa}$. The orbit of the T Tau S system around T Tau N is poorly constrained by the existing observations and can be fit with orbits, ranging from a nearly circular orbit with a period of 475 years to highly eccentric orbits with periods up to $2.7 \times 10^{4}$ years.

$\mathrm{T}$ Tau Sa appeared 0.92 mag brighter than $\mathrm{T} \mathrm{Tau} \mathrm{Sb}$ in the CntK2 image, which is in contrast to the latest $K$-band brightness ratio measurements from January 2014 (Schaefer et al. 2014), where $\mathrm{Sb}$ was brighter than $\mathrm{Sa}$. This photometric variability is similar to the behaviour shown prior to 2006 (Beck et al. 2004; Duchêne et al. 2005), and thus demonstrates that the period of lesser variability, when $\mathrm{Sa}$ and $\mathrm{Sb}$ had comparable brightnesses between 2006-2013, has come to an end. The brightness ratio $\mathrm{H}_{2} / \mathrm{CntK} 2$ is 1.90 , very high for $\mathrm{T}$ Tau $\mathrm{N}$, demonstrating the presence of strong $\mathrm{H}_{2} 1-0 \mathrm{~S}(1)$ emission close to this component. Previous studies found $\mathrm{H}_{2}$ emission around $\mathrm{T}$ Tau $\mathrm{N}$ and $\mathrm{S}$ Gustafsson et al. (2010), which our observations also confirm.

Acknowledgements. The authors would like to thank the SPHERE Science Verification team for their support in obtaining the observations. This work was supported by the Momentum grant of the MTA CSFK Lendület Disc Research Group, and the Hungarian Research Fund OTKA grant K101393.

\section{References}

Akeson, R. L., Koerner, D. W., \& Jensen, E. L. N. 1998, ApJ, 505, 358 Baraffe, I., Chabrier, G., Allard, F., \& Hauschildt, P. H. 1998, A\&A, 337, 403 Beck, T. L., Schaefer, G. H., Simon, M., et al. 2004, ApJ, 614, 235

Duchêne, G., Ghez, A. M., \& McCabe, C. 2002, ApJ, 568, 771

Duchêne, G., Ghez, A. M., McCabe, C., \& Ceccarelli, C. 2005, ApJ, 628, 832

Duchêne, G., Beust, H., Adjali, F., Konopacky, Q. M., \& Ghez, A. M. 2006, A\&A, 457, L9

Furlan, E., Forrest, W. J., Watson, D. M., et al. 2003, ApJ, 596, L87

Gustafsson, M., Kristensen, L. E., Kasper, M., \& Herbst, T. M. 2010, A\&A, 517, A19

Hogerheijde, M. R., van Langevelde, H. J., Mundy, L. G., Blake, G. A., \& van Dishoeck, E. F. 1997, ApJ, 490, L99

Johnston, K. J., Gaume, R. A., Fey, A. L., de Vegt, C., \& Claussen, M. J. 2003, AJ, 125,858

Johnston, K. J., Fey, A. L., Gaume, R. A., Claussen, M. J., \& Hummel, C. A. 2004, AJ, 128, 822

Kasper, M. E., Feldt, M., Herbst, T. M., et al. 2002, ApJ, 568, 267

Köhler, R., Ratzka, T., Herbst, T. M., \& Kasper, M. 2008, A\&A, 482, 929

Loinard, L., Mioduszewski, A. J., Rodríguez, L. F., et al. 2005, ApJ, 619, L179

Maihara, T., \& Kataza, H. 1991, A\&A, 249, 392

Nisenson, P., Stachnik, R. V., Karovska, M., \& Noyes, R. 1985, ApJ, 297, L17

Pál, A. 2012, MNRAS, 421, 1825

Schaefer, G. H., Simon, M., Beck, T. L., Nelan, E., \& Prato, L. 2006, AJ, 132, 2618

Schaefer, G. H., Prato, L., Simon, M., \& Patience, J. 2014, AJ, 147, 157

Smith, K., Pestalozzi, M., Güdel, M., Conway, J., \& Benz, A. O. 2003, A\&A, 406, 957

van Boekel, R., Juhász, A., Henning, T., et al. 2010, A\&A, 517, A16

van den Ancker, M. E., Wesselius, P. R., Tielens, A. G. G. M., van Dishoeck,

E. F., \& Spinoglio, L. 1999, A\&A, 348, 877

White, R. J., \& Ghez, A. M. 2001, ApJ, 556, 265

Page 5 is available in the electronic edition of the journal at http://www . aanda.org 
T Tau N - S

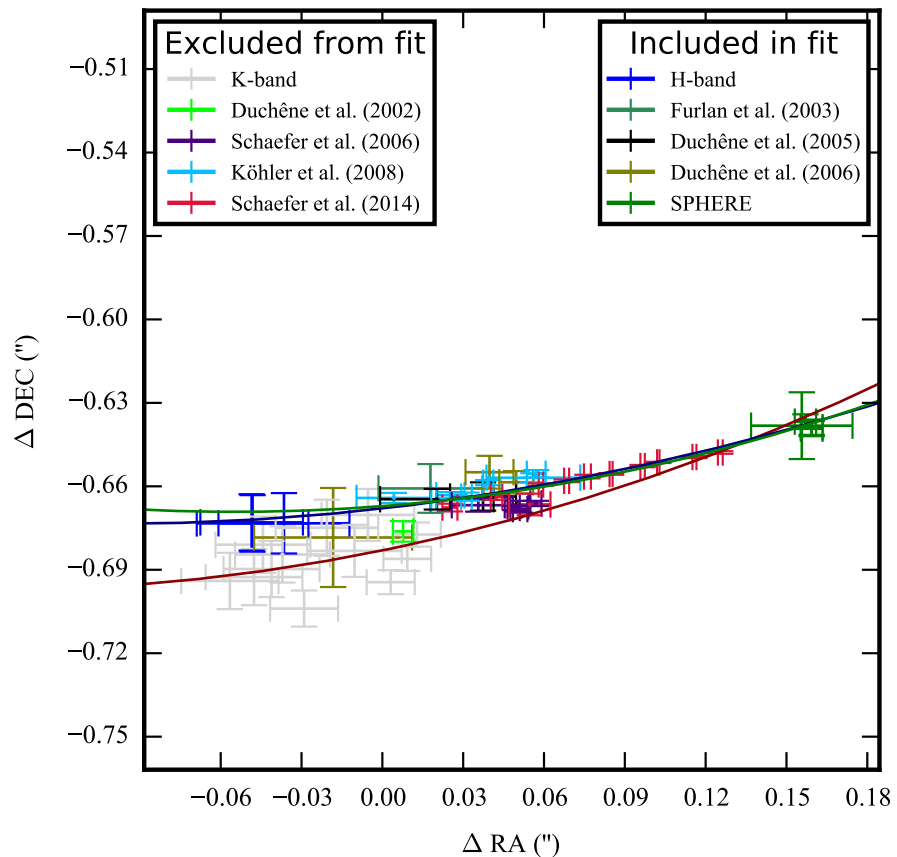

$\mathrm{T}$ Tau $\mathrm{Sa}-\mathrm{Sb}$

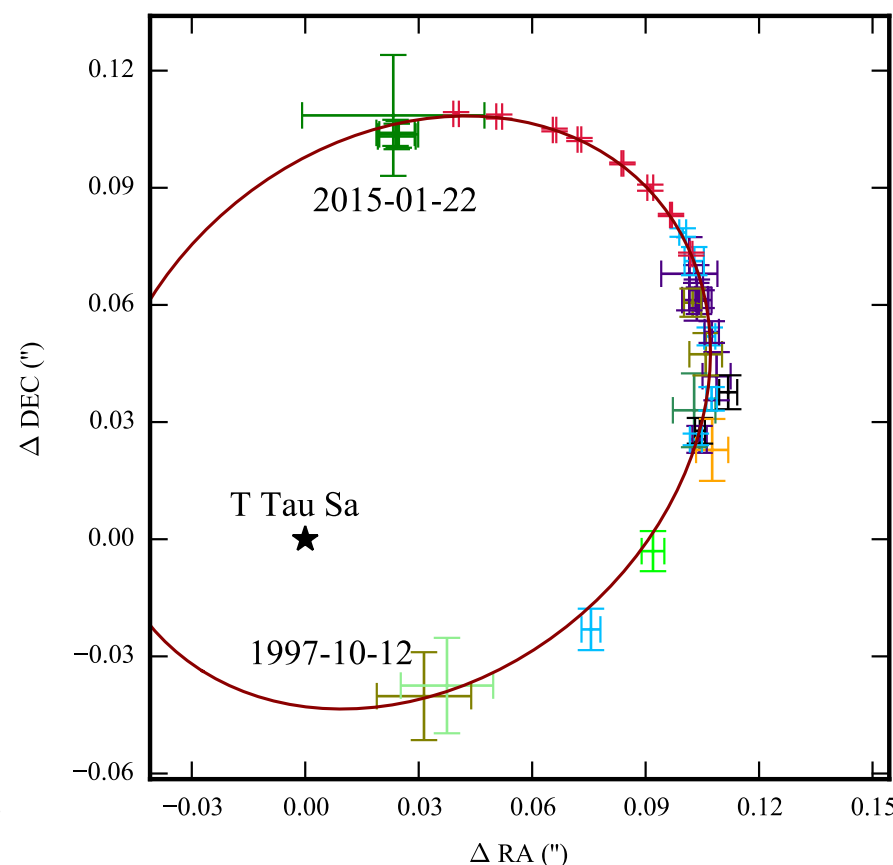

Fig. 3. Left: epochs of T Tau S around T Tau N. The coordinates are relative to T Tau N. Observations in which the Sa/Sb pair is not resolved are shown in either light grey or blue. The coloured markers show the resolved observations, in which the centre of mass was calculated from the position of Sa and Sb. The red line shows the orbit calculated by Köhler et al. (2008). The dark blue and green lines shows two possible orbital fits with the extreme periods of $2.7 \times 10^{4}$ and 475 years, respectively. Right: epochs of T Tau Sb around T Tau Sa. The coordinates are relative to T Tau Sa. The red line shows the orbit calculated by Schaefer et al. (2014).

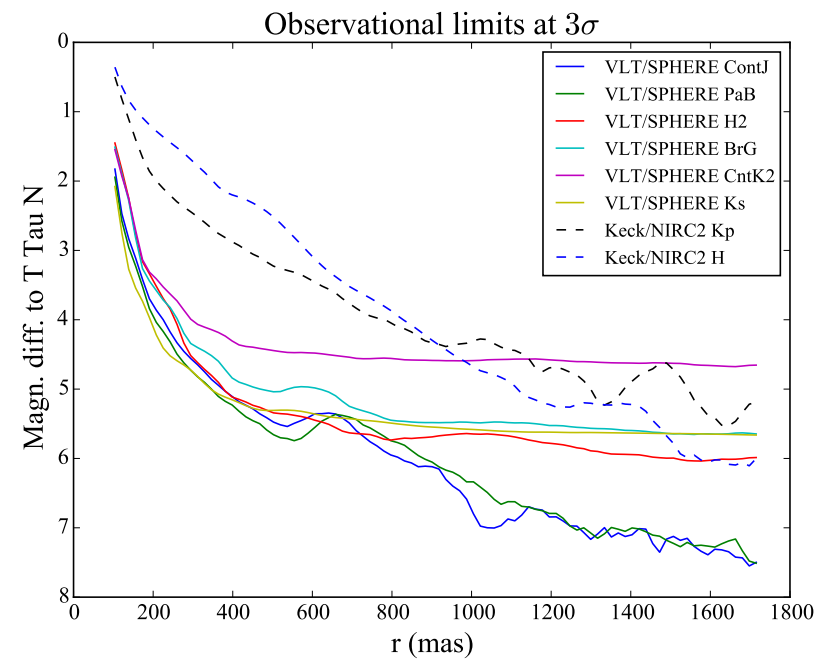

Fig. 4. Detection limits of $3 \sigma$ for point-like sources near $\mathrm{T}$ Tau $\mathrm{N}$ in the classical imaging IRDIS mode of VLT/SPHERE and Keck/NIRC2. The bumps in the SPHERE data at $\approx 250$ and $\approx 600$ mas are the wings of the PSF from T Tau N. The Keck/NIRC2 data is obtained from the Keck Observatory Archive, from projects identified as N26N2 for the $H$-band and 2010B for the $K$-band data.

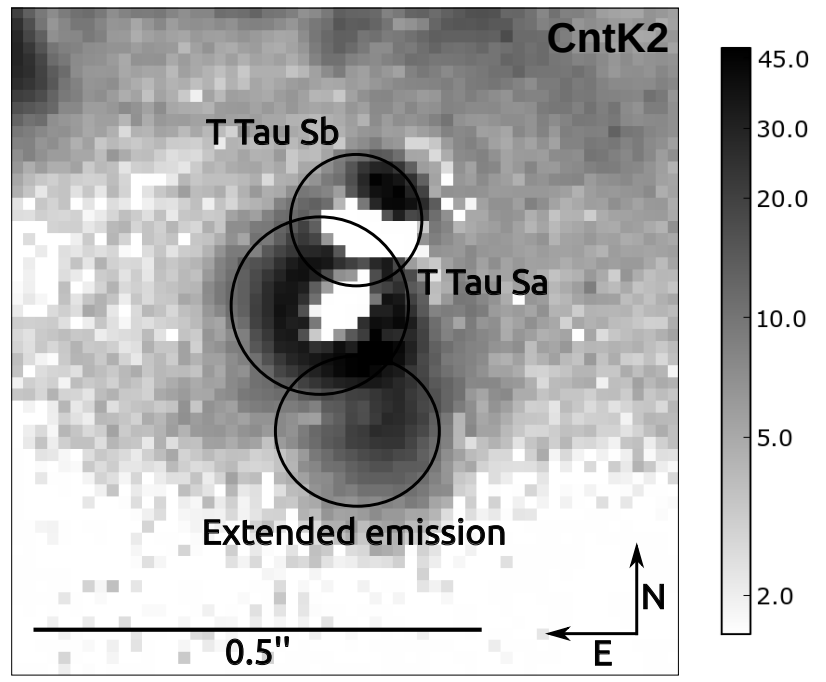

Fig. 5. Extended emission south of $\mathrm{T}$ Tau Sa. Both $\mathrm{T}$ Tau Sa and $\mathrm{Sb}$ were subtracted using T Tau N as a PSF reference scaled to the peak of $\mathrm{T}$ Tau Sa and Sb, respectively. The leftover artefacts are due to the Airy rings apparent around all three components, which cannot be totally eliminated because of the closeness of $\mathrm{T} \mathrm{Tau} \mathrm{Sa}$ and $\mathrm{Sb}$. The leftover residuals have an amplitude of $2.4 \%$ and $6.6 \%$ of the peak fluxes of $\mathrm{T}$ Tau $\mathrm{Sa}$ and $\mathrm{Sb}$, respectively. 